\title{
Requerimientos para el diseño de la experiencia de inmersión en laboratorios virtuales
}

\section{Resumen}

Los laboratorios virtuales son medios muy efectivos en el desarrollo de competencias profesionales. En su desarrollo se suele mimetizar la realidad en busca de una mejor experiencia de inmersión con el diseño de ambientes e interfaces hiperrealistas, lo que redunda en una gran complejidad y altos costos asociados a su implementación. El presente estudio propone un grupo de requerimientos a considerar en el diseño y producción de laboratorios virtuales, con foco en la experiencia de inmersión en contextos no inmersivos. Estos requerimientos consideran el diseño desde una perspectiva holística a favor de una experiencia de inmersión que considere dimensiones como la autenticidad didáctica, externa e interna en correspondencia con su relevancia, apego a la realidad y coherencia. Para ello se llevan a cabo dos ciclos de investigación. En el primero se sistematizan tres proyectos de investigación en el desarrollo de laboratorios virtuales y se definen los requerimientos. Estos se someten a criterio de expertos en la segunda fase a través del método Delphi, lo que permite mejorar y validar la propuesta. Se llega, finalmente, a la definición de 22 requerimientos de diseño que propician la experiencia de inmersión en el desarrollo de laboratorios virtuales, los que pueden resultar de gran utilidad para desarrolladores y docentes vinculados tanto a su producción como a su aplicación educativa.

\author{
Ariane Alvarez Alvarez \\ Doctora en Ciencias de la Educación \\ Universidad Mayor, Santiago de \\ Chile, Chile. \\ Correo electrónico: \\ ariane.alvarez@umayor.cl \\ ๑1 orcid.org/0000-0002-0337-947X \\ Google Scholar \\ Juan Francisco Cabrera \\ Ramos \\ Doctor en Ciencias de la Educación \\ Universidad Católica de Temuco, \\ Temuco, Chile \\ Correo electrónico: jcabrera@uct.cl \\ ๑ orcid.org/0000-0002-1920-2230 \\ Google Scholar
}

Recibido: marzo 29 de 2019 Aprobado: abril 28 de 2020

Palabras clave: aprendizaje, diseño, inmersión, laboratorio virtual, realismo. 


\section{Requirements for the design of the immersion experience in virtual laboratories}

\begin{abstract}
Virtual laboratories are effective means in the development of professional skills. In their development, reality is ususlly mimicked in search of a better immersion experience with the design of hyper-realistic environments and interfaces which results in great complexity and high costs associated with its implementation. This study proposes a group of requirements to be considered in the design and production of virtual laboratories with a focus on the immersion experience in non-immersive contexts. These requirements consider design from a holistic perspective in favor of an immersion experience that considers dimensions such as didactic, external and internal authenticity, in correspondence with its relevance, attachment to reality and coherence. Two research cycles were carried out. In the first cycle, three research projects in the development of virtual laboratories were systematized and the requirements were defined. In the second cycle, the requirements were submitted to expert judgment through the Delphi method which allowed the proposal to be improved and validated. Finally, the definition of 22 immersion experience design requirements in the development of virtual laboratories was proposed. The result is particularly useful for developers and educators in designing learning experiences.
\end{abstract}

Key words:

learning, design, immersion, virtual laboratory, realism. 


\section{Introducción}

La literatura científica muestra un amplio número de contribuciones relacionadas con el desarrollo y uso educativo de los laboratorios virtuales (LV). Se hace énfasis en su carácter de sustitutos de laboratorios reales o remotos (Morcillo Ortega, 2008), en su cualidad de simulación de la realidad, en sus posibilidades de interacción (Dormido Bencomo, Sánchez Sánchez, \& Morilla García, 2000; Monge-Nájera, Rivas, \& Méndez-Estrada, 2002) y en su carácter de entornos virtuales que permiten la socialización y gestión en el proceso de aprendizaje.

Como objeto de aprendizaje, el laboratorio virtual posee una relación dinámica con los demás componentes del proceso de enseñanza aprendizaje desde su dimensión mediadora. Su efectividad, por tanto, está comprometida con la coherencia de su inserción en la propuesta didáctica, con la proyección pedagógica implícita, la forma en que soporta los métodos y propicia la evaluación. Un laboratorio virtual será efectivo en la medida que responda a las exigencias pedagógicas y ofrezca flexibilidad a las condiciones del proceso de enseñanza-aprendizaje.

Los laboratorios virtuales brindan la posibilidad de experimentar a través de una interfaz gráfica, pueden ser usados en cualquier lugar y momento y permiten al estudiante repetir la experiencia de aprendizaje a voluntad (Calvo, Zulueta, Gangoiti, \& López, 2008; Monge Nájera \& Méndez Estrada, 2007), lo cual aumenta el acceso a experiencias de aprendizaje de calidad. Otro factor determinante resulta de que el estudiante no se exponga a accidentes durante su uso y que no se requiera de la presencia física de personal especializado de soporte a la práctica. 
Según el grado en que reproducen la realidad y su interactividad, es común la referencia a que las prácticas de laboratorio en entornos virtuales pueden reemplazar a los laboratorios tradicionales, e incluso al trabajo práctico del estudiante en escenarios reales (Zacharia \& Olympiou, 2011). Ello se sustenta en la riqueza y suficiencia de estos medios para modelar escenarios auténticos de aprendizaje.

En el análisis de la literatura se encuentran dos tendencias en cuanto a la representación de la realidad en laboratorios virtuales: realista y abstracta. La realista se centra en el mimetismo con que se reproduce la apariencia y comportamiento de artefactos y fenómenos y en ella se consideran las formas, texturas, color, el factor escala, la atmósfera, las relaciones espaciales, los sonidos propios de los artefactos y del ambiente. La representación abstracta, por su parte, se sustenta en símbolos que constituyen convenciones operativas. Desde ambas posiciones, la inmersión es una cualidad clave en la relevancia didáctica (Couture, 2004; Francis \& Couture, 2003; Hennessy \& O'Shea, 1993), la que se considera una propiedad objetiva de un ambiente o sistema, orientada a la creación de la sensación de presencia (Slater, 2004; Dalgarno \& Lee, 2010).

La sensación de estar inmerso, cuando se hace uso de una práctica de laboratorio virtual, depende de una configuración compleja de factores, donde destaca la credibilidad (Couture, 2004), marcada por el grado de realismo de la propuesta (Piassentini \& Occelli, 2012).

La estrategia de diseño de realismo con base en la representación mimética de la realidad puede llegar a ser un camino sumamente costoso. Por otra parte, los resultados variarán según el contexto. La teoría de los affordances sugiere cómo la sensación de inmersión depende tanto del ambiente como del observador. La percepción es condicionada por la subjetividad y varía en 
dependencia del sujeto y sus expectativas, permeadas en buena medida por su experiencia (Gibson, 2014).

Couture (2004) y Elliott (1983) establecen los criterios de posibilidad, plausibilidad y existencia como base de los juicios de verosimilitud. Ello tiene implicaciones en la manera en que se concibe una práctica realista y en cómo se mide dicho constructo. Por su parte, Alessi (1988) establece que la relación entre la fidelidad de la simulación y los resultados de aprendizaje no es lineal, por lo que deben considerarse otros factores en el diseño de la inmersión con fines de aprendizaje. Francis \& Couture (2003) y más tarde Couture (2004) Ilevan a cabo estudios sobre la credibilidad y el realismo de un laboratorio virtual basado en simulación y establecen consideraciones sobre cómo conseguir resultados que se perciban realistas. Se destacan las ventajas de la fotografía por sobre la modelación, la que puede ser valorada como una forma económicamente viable de representación mimética de la realidad. Sobre ello, Gonçalves et al. (2010) plantean que la autenticidad de una simulación depende de su autenticidad interna (coherencia), su autenticidad externa (apego a la realidad) y la autenticidad didáctica (relevancia).

Estudios comparados sobre laboratorios basados en fotografías y en esquemas, muestran que los estudiantes perciben más realistas los laboratorios basados en fotos (Ozdemir, Ustun, \& Bagriyanik, 2017). Ello se asocia a sus niveles de concreción y resolución. Aun cuando se valora que en ese resultado pueden estar influyendo las carencias del otro medio utilizado en la comparación, se concuerda en que los estudiantes perciben más realistas aquellas interfaces con menor carga simbólica.

En la experimentación con realidad virtual inmersiva, existe evidencia de que se logra la inmersión, sin embargo, no se consiguen los niveles de aprendizaje pretendidos debido a la sobrecarga y a la distracción que genera este tipo de 
entorno (Makransky, Terkildsen, \& Mayer, 2017). Ello sugiere que la inmersión debe diseñarse cuidadosamente y que un mayor despliegue tecnológico no necesariamente lleva a un mejor aprendizaje.

En cuanto al dimensionamiento y medición del realismo, se encuentran modelos de indicadores de realismo percibido en el contexto de videojuegos (Ribbens, 2013). Sus componentes son el realismo de simulación, la libertad de elección, la penetración perceptual, el realismo social, la autenticidad y participación del personaje. En este sentido resulta interesante el estudio de las dimensiones del realismo en la simulación de juegos digitales (Ribbens, Malliet, Van Eck, \& Larkin, 2016), donde se establece una definición dimensional de realismo y se elabora y valida un instrumento para su medición. Aunque dicha propuesta resulta interesante, no es aplicable al desarrollo de laboratorios virtuales, dada la finalidad educativa de este último. En esta misma dirección, Witmer \& Singer (1998) proponen y validan un cuestionario para medir la presencia en entornos virtuales a partir de factores de control, sensoriales, de distracción y de realismo. Dicho resultado, igual que el anterior, no ofrece soluciones concretas al campo de diseño de laboratorios virtuales, pero constituyen un punto de partida interesante.

Ahmed \& Hasegawa (2014) proponen un modelo de diseño instruccional y los criterios para el diseño y desarrollo de laboratorios virtuales en línea. Este resultado se enfoca en los elementos pedagógicos de la propuesta y en los roles y procedimientos para el desarrollo de laboratorios virtuales, aspecto que igualmente se toma en cuenta.

\section{Métodos}

A partir de la constatación de la complejidad del diseño de la sensación de inmersión en los laboratorios virtuales y el alto costo que implica la producción 
de los escenarios hiperrealistas, se plantea la siguiente pregunta: ¿cómo propiciar la experiencia inmersiva en laboratorios virtuales en contextos no inmersivos? Se considera que la respuesta a esta pregunta debe darse además en un escenario en el que se priorice la racionalidad técnica y didáctica de la propuesta, de manera que se puedan lograr laboratorios virtuales a un costo razonable y que ello conlleve a que se puedan estructurar equipos de desarrollo de laboratorios virtuales como parte de proyectos de soporte en instituciones educativas.

Para responder a esta pregunta se establece como objetivo: modelar requerimientos para el diseño de la experiencia de inmersión en la producción de laboratorios virtuales en contextos no inmersivos. Dicha propuesta se concibe con centro en el diseño y orientado a la autenticidad pedagógica, la externa e interna. Se considera la complejidad implícita en el acto de percepción de realismo, influenciada tanto por aspectos personológicos como por particularidades del proceso de enseñanza aprendizaje en que se inserta el laboratorio virtual.

El estudio se lleva a cabo en dos etapas: un estudio inicial, de la que se obtiene una primera propuesta de requerimientos y una segunda etapa de consulta a expertos que se desarrolla en tres fases.

\section{Estudio inicial (etapa 1)}

Se lleva a cabo un estudio de casos con enfoque auto etnográfico (Atkinson, 2006; Blanco, 2012; Ellis, 1999; Unluer, 2012) bajo el paradigma interpretativo, orientado a la extracción de conocimientos sobre la percepción de realismo en tres proyectos de desarrollo de laboratorios virtuales y sobre la virtualización de 12 prácticas de laboratorio. 
Los casos corresponden a ciclos de desarrollo y prueba de laboratorios virtuales. Se utiliza la técnica de análisis de contenido desde una perspectiva histórico-lógica sobre los informes de proyecto, las narrativas de los autores desde su rol de participantes comprometidos, las encuestas aplicadas a docentes, estudiantes y equipos de desarrollo a través de los diferentes casos y se contrasta con los hallazgos en la literatura especializada.

En esta etapa se obtiene una propuesta de 20 requerimientos para el diseño de la experiencia de inmersión en el desarrollo de laboratorios virtuales.

\section{Consulta a expertos (etapa 2)}

Entre los criterios de inclusión para la selección de expertos se consideraron la experiencia en el diseño y uso de laboratorios virtuales, el contar con publicaciones científicas, la disponibilidad temporal y la motivación por participar en el proceso.

Se envió el cuestionario a un total de 28 expertos. Se comprometieron 19 expertos con el estudio y completaron los datos solicitados y de ellos 16 fueron considerados por tener un coeficiente de competencia aceptable $(K>=0.8)^{1}$. Los expertos se mantuvieron estables a través de las tres rondas.

En la consulta a expertos, se consideraron esenciales los siguientes criterios: (1) conformidad de los expertos con la propuesta, (2) consenso entre expertos y (3) estabilidad de grupo en las respuestas entre fases. El proceso se llevó a cabo como consulta individual a través de internet.

${ }^{1}$ El valor de K se calculó como el promedio entre coeficiente de conocimiento del experto sobre su valoración propia y el fundamento de su competencia a partir de la valoración de su trayectoria. 


\section{Fase 1. Primera ronda de trabajo con expertos}

En esta fase se propone el enriquecimiento y valoración de la propuesta por expertos. Estos evalúan los requerimientos obtenidos en la primera fase de trabajo. Para ello se les solicitó que hicieran sugerencias de ajuste y más tarde, en el mismo instrumento, se les pidió que aportaran otros requerimientos no considerados en la propuesta.

Como insumo, los expertos reciben en esta fase una lista de requerimientos densamente argumentados, notas de campo y literatura que sustenta la propuesta, así como ejemplos concretos sobre cómo se ha visto el requerimiento en procesos anteriores.

\section{Fases 2 y 3: segunda y tercera rondas de trabajo con expertos}

En la segunda y tercera ronda se solicita a expertos que valoren una nueva propuesta de requerimientos, donde califican cada recomendación en una escala de uno a siete ( $1=$ totalmente inconforme; $7=$ totalmente conforme). $\mathrm{Al}$ igual que en la primera ronda se les facilita un espacio para sugerencias o propuestas de cambio. En ambas rondas se les entrega informes ejecutivos con base en estadística descriptiva.

Se consideró como criterio de conformidad con la propuesta el que se obtuviera una media (acotada al 25\%) que fuera igual o mayor a 5 en la escala de 1 a 7. La acotación excluye las dos puntuaciones más bajas y las dos puntuaciones más altas a los propósitos de reducir sesgos en los criterios de los expertos. Los requerimientos sobre los que se obtuvo una valoración como conformes según la respuesta de los expertos y sobre los que se alcanzó consenso en la segunda ronda no se ajustaron nuevamente, sin embargo, se consideraron en la siguiente consulta a propósito de la búsqueda de estabilidad en la respuesta. 
Se consideró el consenso con base en cada requerimiento sobre un rango intercuartil de 1 o menor y como criterio de estabilidad que no existieran diferencias estadísticamente significativas en las respuestas de expertos en dos rondas sucesivas a través de la aplicación de la prueba de los rangos con signos de Wilcoxon (von der Gracht, 2012). Esta última prueba es una alternativa no paramétrica a la aplicación de $\mathrm{T}$ de Student para muestras relacionadas, valioso en este caso por el tamaño reducido de la muestra a analizar.

Al final de la tercera ronda se estimó que los niveles de conformidad eran aceptables, que existía consenso y estabilidad de grupo en las valoraciones otorgadas. Sobre esa base y tomando en cuenta la disponibilidad de tiempo de los expertos participantes, se decide concluir la consulta a expertos.

\section{Resultados}

\section{Recomendaciones para el diseño de la experiencia de inmersión en laboratorios virtuales tras fase 1 del estudio.}

La experiencia de inmersión constituye un fenómeno complejo, influenciado por múltiples factores, por lo que contar con un grupo de requerimientos de diseño a su favor no se limita a una lista de chequeo para desarrolladores de los laboratorios virtuales. El peso de los requerimientos de diseño en la toma de decisiones asociada a la conceptualización y desarrollo de alternativas de solución resulta notable, redundando en un menor costo y complejidad de las propuestas para el logro de una experiencia de inmersión adecuada. Los requerimientos permitirán, adicionalmente, evaluar el potencial de las propuestas de laboratorio virtual para el logro de la experiencia de inmersión, tanto dentro de un proceso de desarrollo como en la determinación para la adquisición de laboratorios virtuales o la investigación en didáctica por parte de las instituciones educativas. 
Los requerimientos relacionados en la siguiente tabla son el resultado del proceso de análisis inicial y su contraste con la literatura científica. Dichos requerimientos fueron organizados a partir de tres dimensiones: autenticidad didáctica, autenticidad externa y autenticidad interna, en correspondencia con su relevancia, apego a la realidad y coherencia (Gonçalves et al., 2010). En la tabla 1 se muestra la síntesis de los requerimientos obtenidos en la primera etapa de trabajo.

Tabla 1. Propuesta inicial de requerimientos de diseño desde las dimensiones establecidas

\begin{tabular}{|c|c|}
\hline Dimensiones & Requerimientos de diseño \\
\hline $\begin{array}{l}\text { Autenticidad didáctica } \\
\text { (relevancia) }\end{array}$ & $\begin{array}{l}\text { Modelar con centro en el aprendizaje, no en artefactos } \\
\text { Orientación de la práctica con foco en el logro de la competencia profesional } \\
\text { Considerar la experiencia previa del estudiante } \\
\text { Propiciar la motivación }\end{array}$ \\
\hline $\begin{array}{l}\text { Autenticidad externa } \\
\text { (apego a la realidad) }\end{array}$ & $\begin{array}{l}\text { Procurar una interfaz autoevidente y poco invasiva } \\
\text { Simular la realidad, no el laboratorio real } \\
\text { Acotar el acercamiento } \\
\text { Controlar el ritmo } \\
\text { Diseñar preferiblemente entornos 2.5D } \\
\text { Potenciar la retroalimentación sonora realista } \\
\text { Flexibilizar la interacción } \\
\text { Establecer compromisos con el sistema simbólico } \\
\text { Optimizar la representación visual } \\
\text { Implantar el sistema simbólico } \\
\text { Economizar mediadores en la interfaz }\end{array}$ \\
\hline $\begin{array}{l}\text { Autenticidad interna } \\
\text { (coherencia) }\end{array}$ & $\begin{array}{l}\text { Fidelizar el comportamiento funcional } \\
\text { Guardar estado de avance en la práctica } \\
\text { Propiciar interacción multiusuario } \\
\text { Fidelizar la representación temporal } \\
\text { Simplificar la modelación de lo que no desarrolla competencias }\end{array}$ \\
\hline
\end{tabular}

Fuente: elaboración propia. 
En la tabla número 2 se muestran los requerimientos definitivos. En la misma se esclarecen las modificaciones realizadas durante las tres fases de trabajo con expertos. Las propuestas de cambio a los requerimientos se concentraron fundamentalmente en la primera fase. El rango intercuartil, utilizado como criterio de consenso, se calculó sobre el total de las respuestas $(n=16)$, a diferencia de la conformidad que se calculó sobre la media acotada al 25\% $(n=12)$.

Tabla 2. Requerimientos definitivos y resultados de aplicación del método Delphi de expertos

\begin{tabular}{|c|c|c|c|c|c|c|}
\hline \multirow[b]{2}{*}{$\#$} & \multirow[b]{2}{*}{ Requerimientos definitivos } & \multirow{2}{*}{$\begin{array}{l}\text { Estado final con } \\
\text { respecto a la } \\
\text { propuesta inicial }\end{array}$} & \multicolumn{2}{|c|}{ Fase 2} & \multicolumn{2}{|c|}{ Fase 3} \\
\hline & & & $\begin{array}{c}X \\
(25 \%)\end{array}$ & RIQ & $\begin{array}{c}X \\
(25 \%)\end{array}$ & RIQ \\
\hline 1 & $\begin{array}{l}\text { Diseñar el LV tomando como eje la lógica y } \\
\text { dinámicas más factibles para el aprendizaje. }\end{array}$ & Ajustado & 6,33 & 1 & 6,17 & 0,25 \\
\hline 2 & $\begin{array}{l}\text { Orientación de la práctica con foco en el logro } \\
\text { de la competencia profesional. }\end{array}$ & Ajustado & 6,83 & 0,25 & 6,83 & 0,25 \\
\hline 3 & $\begin{array}{l}\text { Simulación del procedimiento experimental } \\
\text { como parte de la ayuda. }\end{array}$ & Derivado & 6,42 & 1 & 6,33 & 1 \\
\hline 4 & $\begin{array}{l}\text { Conocimientos previos como prerrequisito o } \\
\text { contenido de las ayudas. }\end{array}$ & Derivado & 6,75 & 1 & 6,83 & 0,25 \\
\hline 5 & Concebir una práctica de familiarización. & Derivado & 5,75 & 1 & 5,83 & 0,25 \\
\hline 6 & $\begin{array}{l}\text { Reforzar el rol y la responsabilidad profesional en } \\
\text { la fijación de las metas. }\end{array}$ & Ajustado & 6,58 & 1 & 6,66 & 1 \\
\hline 7 & $\begin{array}{l}\text { Procurar una interfaz autoevidente y poco } \\
\text { invasiva. }\end{array}$ & Estable & 6,83 & 0,25 & 6,75 & 0,25 \\
\hline 8 & $\begin{array}{l}\text { Valorar la simulación de los escenarios reales por } \\
\text { sobre la simulación del laboratorio real. }\end{array}$ & Ajustado & 5,17 & 0,75 & 5,5 & 1 \\
\hline 9 & Acotar el acercamiento. & Estable & 5,83 & 0,75 & 5,58 & 1 \\
\hline 10 & Controlar el ritmo. & Estable & 6,25 & 1 & 6,42 & 1 \\
\hline
\end{tabular}




\begin{tabular}{|c|c|c|c|c|c|c|}
\hline \multirow[b]{2}{*}{ \# } & \multirow[b]{2}{*}{ Requerimientos definitivos } & \multirow{2}{*}{$\begin{array}{l}\text { Estado final con } \\
\text { respecto a la } \\
\text { propuesta inicial }\end{array}$} & \multicolumn{2}{|c|}{ Fase 2} & \multicolumn{2}{|c|}{ Fase 3} \\
\hline & & & $\begin{array}{c}X \\
(25 \%)\end{array}$ & RIQ & $\begin{array}{c}X \\
(25 \%)\end{array}$ & RIQ \\
\hline 11 & $\begin{array}{l}\text { Condicionar la representación dimensional a los } \\
\text { propósitos y demandas del laboratorio. }\end{array}$ & Ajustado & 5,25 & 1 & 5,33 & 1 \\
\hline 12 & Potenciar la retroalimentación sonora. & Estable & 6,33 & 1 & 6,17 & 0,25 \\
\hline 13 & Flexibilizar la interacción. & Estable & 6,42 & 1 & 6,33 & 1 \\
\hline 14 & Establecer compromisos con el sistema simbólico. & Estable & 6,5 & 1 & 6,25 & 1 \\
\hline 15 & Optimizar la representación visual. & Estable & 6,25 & 1 & 6,17 & 0,25 \\
\hline 16 & Significar el sistema simbólico. & Ajustado & 6,17 & 0,25 & 6,08 & 0 \\
\hline 17 & $\begin{array}{l}\text { Evitar mediadores en la interfaz dedicados a la } \\
\text { argumentación. }\end{array}$ & Ajustado & 6,08 & 0 & 6 & 0 \\
\hline 18 & Fidelizar el comportamiento funcional. & Estable & 6,92 & 0 & 7 & 0 \\
\hline 19 & Guardar estado de avance en la práctica. & Estable & 5,25 & 1 & 5,17 & 1 \\
\hline 20 & Propiciar interacción multiusuario. & Estable & 5,08 & 0,5 & 5,25 & 1 \\
\hline 21 & Fidelizar la representación temporal. & Estable & 6,25 & 1 & 6,33 & 1 \\
\hline 22 & $\begin{array}{l}\text { Simplificar la modelación de lo que no desarrolla } \\
\text { competencias. }\end{array}$ & Estable & 6,33 & 1 & 6,25 & 1 \\
\hline
\end{tabular}

Fuente: elaboración propia.

En la valoración de correlación entre la segunda y tercera fases, en la búsqueda de estabilidad en la respuesta de los expertos, se aplicó la prueba de los rangos con signos de Wilcoxon, donde no se encontraron diferencias significativas entre ambos resultados $(Z=-.831 ; p=.406)$, por lo que se consideró que la estabilidad de las respuestas de los jueces es muy apropiada. 
Las respuestas de los expertos fueron consideradas íntegramente en la propuesta y los resultados fueron devueltos en las siguientes rondas. Sin embargo, resulta de interés el análisis cuantitativo de las valoraciones de los expertos. Cinco expertos expresan que la propuesta de requerimientos ofrece una opción concreta que debería ser seguida por los desarrolladores de laboratorios virtuales $(1,4,5,8,15$ y 16) y seis argumentaron que es una propuesta esclarecedora sobre cómo actuar convenientemente desde el diseño y que son claves en la estructuración de procesos de desarrollo $(2,3,8,11,14$ y 15). Tres expertos hacen énfasis en la idea de que no es solo una propuesta para diseñadores gráficos, ya que su despliegue considera el trabajo de todo el equipo de desarrollo de laboratorios virtuales (7, 12 y 15).

Aunque tres expertos (3, 5 y 9) plantean que la propuesta brinda una perspectiva utilitaria del diseño, aspecto que no se valora como una crítica, resulta interesante la contraposición a la idea expresada por ellos mismos y por otros expertos sobre el alcance multidimensional de la propuesta.

Se hace énfasis en la relevancia de una propuesta que se oriente a la mejora de la relación costo-beneficio en la sensación de inmersión, aspecto que fue considerado como clave por siete de los expertos (2, 5, 6, 8, 11, 13 y 16). Igualmente se considera la factibilidad general de la propuesta, con énfasis en la dirección de la factibilidad didáctica (10, 11, 14 y 16).

En las observaciones de los expertos se refuerza la idea de que la sensación de inmersión depende, en gran medida, de la componente pedagógica. Sobre el requerimiento 3 comentan: "Es muy variable y difícil de controlar, solo se constata en la aplicación de la práctica" (7), "La falta de experiencia se puede compensar con una buena orientación" (9), "En la inducción de la práctica se debe insistir en los conocimientos previos, en la orientación del laboratorio y en las prácticas de familiarización" (1). Sobre el requerimiento 4: "depende 
tanto del docente como del laboratorio" (8), "puede estar condicionada por múltiples factores ajenos al laboratorio" (4).

Otra idea que destaca en las observaciones en la necesidad de generar vínculos que refuercen la responsabilidad en las tareas profesionales: La colaboración es altamente deseable" (10), "Genera más apego a la realidad. Los profesionales no trabajan solos" (13).

Aunque el requerimiento 6 es reconocido como "una propuesta original" (3) varios expertos alertan sobre la dificultad para representar ambientes naturales, así como para generar la interfaz de interacción (1, 3, 7, 15). Se flexibiliza la propuesta dada la recomendación de "(...) valorarse cada caso, porque la propuesta es muy categórica" (6).

El requerimiento 9 es considerado por algunos expertos como una coyuntura condicionada por la opción tecnológica $(2,5,4,9,11)$ y a partir de las recomendaciones se ajusta la propuesta. Otros apuntan a la necesidad de no ser inflexibles: "En la medida que la interacción con los artefactos requiera recorridos más flexibles serán necesarios los entornos 3D" (7); "Es una decisión que debe tomarse a partir de las particularidades del laboratorio, el requerimiento debe apuntar a la relación entre la demanda de libertad de movimiento del usuario y la flexibilidad del escenario" (16).

Entre los requerimientos mejor valorados destaca el 16: “(...) marca la diferencia entre un laboratorio bueno y uno malo" (2), "La fidelidad en la modelación matemática de los fenómenos es vital" (5).

Se reconoce que el requerimiento 20 implica establecer prioridades en la virtualización según la relevancia en el aprendizaje, lo que demanda de una alta cohesión del equipo de desarrollo. Los expertos Ilaman la atención sobre 
la necesidad de mantener la integralidad de la interfaz $(1,12)$ : "aligerar los escenarios puede ayudar en esta dirección"; "No incluir objetos que no puedan ser manipulados" (16).

\section{Discusión}

Se reconoce que la sensación de inmersión está asociada a las vivencias y características de cada sujeto (Ribbens \& Malliet, 2010; Witmer \& Singer, 1998), por lo que el elemento cultural debe ser tomado en cuenta a la hora de diseñar este tipo de simulaciones con propósito educativo. Esta dimensión queda transversal en la propuesta.

La experiencia inmersiva en un laboratorio virtual debe propiciarse considerando todo el proceso de interacción con el laboratorio, desde la orientación de la actividad. Si el laboratorio virtual se inserta coherentemente en el sistema de medios de la asignatura, bajo una proyección pedagógica adecuada, sus posibilidades de éxito se incrementarán y en ello será más efectiva la experiencia inmersiva.

La sensación de inmersión depende de múltiples factores, entre los que no escapa el contexto y las particularidades del proceso donde se aplican los laboratorios virtuales y la propia naturaleza del laboratorio que se esté desarrollando. Es por ello por lo que los requerimientos de diseño propuestos no constituyen una lista de chequeo, sino consideraciones que deben valorarse en su interrelación y de forma holística.

Se pueden establecer varias fases en una experiencia de aprendizaje con laboratorios virtuales: la orientación, la ejecución y el cierre. Los laboratorios virtuales, en su condición de medios de enseñanza-aprendizaje, están muy condicionados por la proyección pedagógica y la estrategia didáctica desde 
las que se orientan y desarrollan. Obviar esta vinculación puede afectar en buena medida la sensación de inmersión pretendida en la virtualización, de ahí la importancia que los autores le conceden a la autenticidad didáctica. Es deseable que el desarrollo de laboratorios virtuales se inscriba en una proyección desarrolladora del aprendizaje. La autenticidad didáctica condiciona en gran medida el logro de la sensación de inmersión, la que se manifiesta durante la interacción del estudiante con los artefactos (autenticidad externa) y al comportamiento fenomenológico de los mismos (autenticidad interna). Ambos se hacen perceptibles desde la retroalimentación que recibe el estudiante y la naturalidad con que se muestran las consecuencias de su acción.

Los requerimientos propuestos, asociados a autenticidad externa (AE), son coherentes con la teoría de los affordances de James Jerome Gibson. Lo más significativo de esta teoría para la propuesta es la consideración de la dualidad e interdependencia que se establece entre el ambiente y el observador durante la experiencia de interacción. Las affordances se producen por las características del objeto y por las necesidades del individuo en acción (Gibson, 2014). La experiencia del estudiante, en el caso de los laboratorios virtuales, condiciona de forma notable el modo en que percibe las opciones de interacción y ello es directamente proporcional a la sensación de inmersión.

El laboratorio virtual es mediado por tres niveles de affordance, que complejizan la sensación de inmersión: el más básico, asociado al manejo del hardware (teclado, mouse, guantes, etc.), el intermedio, relacionado con la interfaz gráfica o las acciones para acceder y actuar sobre los artefactos (donde se establecen el grado y complejidad de la interacción) y el nivel superior, asociado a la lógica con que operan los artefactos. Un estudiante con amplio dominio del hardware y el software tendrá menos barreras a la inmersión. De igual forma, sus experiencias en productos digitales interactivos pueden generar expectativas que no sean cubiertas por el LV. El modo en que los 
artefactos responden a la acción establece, en buena medida, la credibilidad de la acción. La autenticidad externa, más asociada a la forma en que se interactúa con los artefactos a través de la interfaz gráfica, demanda un alto nivel de simplicidad (Nielsen, 1999). El tercer nivel se establece en la interacción con la práctica de laboratorio, a partir del procedimiento que involucra la tarea de aprendizaje. Las expectativas asociadas al aprendizaje establecen condiciones a favor o en contra de la inmersión, según las particularidades del proceso y del estudiante.

\section{Conclusiones}

En cumplimiento del objetivo de la investigación, se llega a la conclusión de que se puede propiciar el diseño de inmersión en laboratorios virtuales no inmersivos a partir del seguimiento de los 22 requerimientos que se proponen en el cuerpo de este artículo. Dicha propuesta ha sido valorada favorablemente por expertos, por lo que constituye un referente a considerar por equipos de desarrollo de laboratorios virtuales.

Con la propuesta de requerimientos de diseño para el logro de la sensación de inmersión se pretende brindar a los equipos de desarrollo una guía para la toma de decisiones durante la conceptualización, desarrollo y aplicación de laboratorios virtuales. Están dirigidas al equipo, no solo a los diseñadores, puesto que, en buena medida, los requerimientos enfatizan la componente pedagógica implícita.

Se debe resaltar que, aunque la propuesta es el resultado de un análisis minucioso, no tiene la intención de ser una receta universal, por lo que se deberá tomar en consideración según las características de cada contexto. 
Finalmente, los autores destacan la contribución de los expertos durante la validación de la propuesta. Las diferentes perspectivas en el análisis corroboran la complejidad del tema y la necesidad de debatir e investigar más alrededor del desarrollo de los laboratorios virtuales como herramienta de gran utilidad para la enseñanza. Nuevos procesos de aplicación y refinamiento serán necesarios en la búsqueda de universalidad y particularidad según el contexto en que se desarrollen los laboratorios virtuales. Para ello se recomienda que se continúe la investigación teórico-práctica en este sentido, fundamentalmente por la necesidad de contar con referentes claros y expeditos que guíen el desarrollo y uso pedagógico de este útil y complejo medio de enseñanza-aprendizaje.

\section{Conflictos de interés}

No existen conflictos de interés entre los autores. Se reconoce el aporte de investigadores, técnicos, docentes y estudiantes que participaron en los tres proyectos que sirven de base a la propuesta y los aportes individuales de los expertos consultados.

\section{Referencias}

Ahmed, M. E., \& Hasegawa, S. (2014). An instructional design model and criteria for designing and developing online virtual labs. International Journal of Digital Information and Wireless Communications (IJDIWC), 4(3), 355.371. http://dx.doi.org/10.17781/P001289

Alessi, S. M. (1988). Fidelity in the Design of Instructional Simulations. Journal of Computer-Based Instruction, 15(2), 40-47. Retrieved October 16, 2020 from https://www.learntechlib.org/p/170285/.

Atkinson, P. (2006). Rescuing autoethnography. Journal of contemporary ethnography, 35(4), 400-404. https://doi.org/10.1177/0891241606286980 
Blanco, Mercedes. (2012). Autoetnografía: una forma narrativa de generación de conocimientos. Andamios, 9(19), 49-74. Recuperado en 16 de octubre de 2020, de http://www.scielo.org.mx/scielo.php?script=sci_ arttext\&pid=S1870-00632012000200004\&lng=es\&tlng=es.

Calvo, I., Zulueta, E., Gangoiti, U. y López, J. M. (2008). Laboratorios remotos y virtuales en enseñanzas técnicas y científicas. Ikastorratza, 3, 1-21.

Couture, M. (2004). Realism in the design process and credibility of a simulation-based virtual laboratory. Journal of Computer Assisted Learning, 20(1), 40-49. https://doi.org/10.1111/j.1365-2729.2004.00064.x

Dalgarno, B., \& Lee, M. J. (2010). What are the learning affordances of 3-D virtual environments? British Journal of Educational Technology, 41(1), 10-32. https://doi.org/10.1111/j.1467-8535.2009.01038.x

Dormido Bencomo, S., Sánchez Sánchez, J. y Morilla García, F. (2000). Laboratorios virtuales remotos para la práctica a distancia de la automática. XXI Jornadas de Automática, Conferencia plenaria, Sevilla.

Elliott, W. R., Rudd, L. R., \& Good, L. (1983, August). Measuring perceived reality of television: Perceived plausibility, perceived superficiality, and the degree of personal utility. Paper presented at the Association for Education in Journalism and Mass Communication Annual Conference, Corvallis, Oregon.

Ellis, C. (1999). Heartful autoethnography. Qualitative Health Research, 9(5), 669-683. https://doi.org/10.1177/104973299129122153 
Francis, A. \& Couture, M. (2003). Credibility of a simulation-based virtual laboratory: An exploratory study of learner judgments of verisimilitude. Journal of Interactive Learning Research, 14(4), 439-464. Norfolk, VA: Association for the Advancement of Computing in Education (AACE). Retrieved October 17, 2020 from https://www.learntechlib.org/ primary/p/2066/.

Gibson, J. J. (2014). The Ecological Approach to Visual Perception: Classic Edition. Psychology Press. https://doi.org/10.4324/9781315740218

Gonçalves, C., Croset, M. C., Ney, M., Balacheff, N., \& Bosson, J. L. (2010). Authenticity in learning game: how it is designed and perceived. En European Conference on Technology Enhanced Learning (pp. 109-122). Springer.

Makransky, G., Terkildsen, T. S., \& Mayer, R. E. (2017). Adding immersive virtual reality to a science lab simulation causes more presence but less learning. Learning and Instruction. https://doi.org/10.1016/j. learninstruc.2017.12.007

Monge Nájera, Julián, \& Méndez Estrada, Víctor Hugo (2007). Ventajas y desventajas de usar laboratoriosvirtuales en educación a distancia:la opinión del estudiantado en un proyectode seis años de duración. Revista Educación, 31(1), 91-108. [fecha de Consulta 16 de Octubre de 2020]. ISSN: 0379-7082. Disponible en: https://www.redalyc.org/articulo. oa? id=440/44031106

Monge-Nájera, J., Rivas, M., \& Méndez-Estrada, V. H. (2002). La evolución de los laboratorios virtuales durante una experiencia de cuatro años con estudiantes a distancia. En XI Congreso Internacional sobre Tecnología y Educación a Distancia (Vol. 5). 
Morcillo Ortega, J. G. (2008). Los laboratorios virtuales aplicados a la biología en la enseñanza secundaria. Una evaluación basada en el modelo "CIPP" (PhD Thesis). Universidad Complutense de Madrid.

Nielsen, J. (1999). Designing Web Usability: The Practice of Simplicity. Thousand Oaks, CA, USA: New Riders Publishing.

Ozdemir, E., Ustun, U., \& Bagriyanik, K. E. (2017). How credible are the virtual laboratories? A comparison of the photographic and non-photographic virtual experiments about resistivity. Presentado en ESERA 2017 Conference (p. 3).

Piassentini, M. J., \& Occelli, M. (2012). Caracterización de Laboratorios Virtuales para la enseñanza de la Ingeniería Genética. En Garcia L., Buffa L. M., Liscovsky, I., Malin Vilar T. G. (Comps.) Memorias de las X Jornadas Nacionales y $V$ Congreso Internacional de Enseñanza de la Biología (pp. 671-676).

Ribbens, W. (2013). Perceived game realism: A test of three alternative models. Cyberpsychology, Behavior, and Social Networking, 16(1), 31-36. https://doi.org/10.1089/cyber.2012.0212

Ribbens, W., \& Malliet, S. (2010). Perceived digital game realism: A quantitative exploration of its structure. Presence: Teleoperators and Virtual Environments, 19(6), 585-600. https://doi.org/10.1162/pres_a_00024

Ribbens, W., Malliet, S., Van Eck, R., \& Larkin, D. (2016). Perceived realism in shooting games: Towards scale validation. Computers in Human Behavior, 64, 308-318. https://doi.org/10.1016/j.chb.2016.06.055

Slater, M. (2004). How colorful was your day? Why questionnaires cannot assess presence in virtual environments. Presence: Teleoperators \& Virtual Environments, 13(4), 484-493. https://doi.org/10.1162/1054746041944849 
Unluer, S. (2012). Being an Insider Researcher While Conducting Case Study Research. The Qualitative Report, 17(29), 1-14. Retrieved from https://nsuworks.nova.edu/tqr/vol17/iss29/2

Von der Gracht, H. A. (2012). Consensus measurement in Delphi studies. Technological Forecasting and Social Change, 79(8), 1525-1536. https://doi.org/10.1016/j.techfore.2012.04.013

Witmer, B. G., \& Singer, M. J. (1998). Measuring presence in virtual environments: A presence questionnaire. Presence, 7(3), 225-240. https://www.mitpressjournals.org/doi/abs/10.1162/105474698565686

C.Zacharia., \& Olympiou, G. (2011). Physical versus virtual manipulative experimentation in physics learning. Learning and Instruction. 21(3), 317-31. https://doi.org/10.1016/j.learninstruc.2010.03.001

Como citar: Alvarez, A y Cabrera, J. F. (2020). Requerimientos para el diseño de la experiencia de inmersión en laboratorios virtuales. Revista KEPES, 17(22), 277-299. https://doi.org/10.17151/kepes.2020.17.22.11 